EMSP FINAL REPORT

U.S. Department of Energy

\title{
Adsorption /Membrane Filtration as a Contaminant Concentration and Separation Process for Mixed Wastes and Tank Wastes
}

Principal Investigator: Mark M. Benjamin

Department of Civil and Environmental Engineering, Box 352700, University of Washington, Seattle WA, 98195-2700 tel. (206) 543 7645, e-mail markbenj@u.washington.edu

Co-Investigator: Gregory V. Korshin

Department of Civil and Environmental Engineering, Box 352700 , University of Washington, Seattle WA, 98195-2700

tel. (206) 543 2394, e-mail korshin@u.washington.edu

Project Number: 55146

Grant Number DE-FG07-96ER62313

Grant Project Officers

Project Duration: October 1996 to October 1999 


\section{Table of Contents}

Executive Summary




\section{Executive Summary}

This project was conducted to evaluate novel approaches for removing radioactive strontium (Sr) and cesium (Cs) from the tank wastes stored at the Hanford Nuclear Reservation in Hanford, WA. Although those two elements are present in very low concentrations in the wastes on a mass basis, they account for the overwhelming majority (>99\%) of the radioactivity in the wastes. The same waste solutions contain many other potentially toxic chemicals, especially heavy metals, that require treatment. However, the available techniques for treating and ultimately disposing of the non-radioactive contaminants are largely ineffective when applied to Sr and Cs removal. Separation of the Sr and Cs from the rest of the waste stream is therefore desirable both as a direct approach for treating those contaminants and also as a preparatory step for treating the non-radioactive contaminants of concern. The challenges associated with these separation steps are that (1) $\mathrm{Sr}$ and Cs are not contaminants from any common industrial process, so their treatment has not been widely studied in the past, i.e., there is no historical data base for successful treatment of these types of waste, and (2) the remaining composition of the waste, especially the fantastically high concentration of strong base that it contains, is also highly unusual, so that its effect on treatment processes is uncertain.

The technique currently envisioned by DOE for treating the $\mathrm{Sr}$ is adsorption of $\mathrm{Sr}$ onto the surfaces of specially prepared inorganic solids (titanium oxides doped with silicates) that are added to the solutions. The $\mathrm{Sr}$ is expected to become associated with the solids, which can then be separated from the solution by settling and filtration, and vitrified. This process is problematic because the particles are used only once and then must be disposed of by a very expensive process. In addition, it requires extensive handling and processing of the particles after they are loaded with radioactive $\mathrm{Sr}$, and it requires very efficient separation of the solids from solution, which can be problematic. 
The bulk of the Sr removal research conducted as part of this project investigated adsorption of Sr onto a novel adsorbent known as iron-oxide-coated sand (IOCS). This adsorbent has the advantages that it can be packed in a column through which the contaminated water flows, so that no subsequent solid/liquid separation is needed: the water exits the column and the IOCS stays behind. (This physical arrangement is not feasible with the titanium oxides, because they are small particles that do not allow water to flow through them at an appreciable rate.) A second advantage is that the IOCS can be regenerated in place. That is, once the IOCS becomes loaded with $\mathrm{Sr}$ from large volumes of waste, the $\mathrm{Sr}$ can be released into a small volume of regenerant solution (consisting of a small concentration of acid in water), and the IOCS can be reused. This solution can be evaporated to a very small volume and, presumably, vitrified. Thus, the need to dispose of a large mass of solids with the contaminant of concern is eliminated.

In the research, non-radioactive $\mathrm{Sr}$ was added to simulated tank solutions and was treated at bench-scale in columns packed with IOCS. The system operated very efficiently, achieving $>99 \%$ Sr removal over much of the test period, and >97\% removal over the entire test period, except for a brief span during an equipment failure. Four columns of IOCS were used, and each column was regenerated 16 times over the course of 30 weeks of testing, with no evidence of any deterioration. Solutions containing organic compounds that have been shown to interfere with $\mathrm{Sr}$ adsorption could be treated effectively by addition of $\mathrm{Ca}$ prior to treatment. Discussions with DOE personnel suggest that $\mathrm{Ca}$ addition might lead to some practical problems with treatment, but model simulations suggest that replacement of the $\mathrm{Ca}$ with $\mathrm{Mg}$ should have the same beneficial effects, without the practical drawbacks.

The second major focus of the work was on the removal of cesium. Since the chemistries of strontium and cesium have little commonality, different materials (namely, cesium scavengers known as hexacyanoferrates, $\mathrm{HCFs}$ ) were employed in these tests. HCFs are 
extremely selective to cesium even in the presence of overwhelmingly higher concentrations of sodium, potassium, calcium and aluminum, all of which are present at high concentration in the Hanford tank wastes. Similarly to many strontium scavengers, the HCFs are normally used only once and then disposed of. However, HCFs cannot be processed and vitrified simultaneously with other radioactive solids, and this has created long-term storage problems.

In the research, we explored ways to regenerate and reuse Cs-saturated HCFs. Our approach was based on the electrochemical (EC) control of ion-exchange interactions between Cs and HCFs. This technology is henceforth referred to EIX. Cesium can be retained by or expelled from HCF if the iron constituting the backbone of the HCF is reduced or oxidized, respectively. The oxidation and/or reduction of the iron can, in some cases, be accomplished chemically, but only via the use of highly unstable and dangerous chemicals (e.g., conc entrated nitric acid, hydrazine, hydroxylamine). By contrast, multicycle regeneration of HCF can, in theory, be accomplished using EIX, in which case no toxic or dangerous chemicals are used, because the entire process is controlled electronically.

The major challenge to implementing EIX is the need to develop high-performance HCFbased electrodes. In this project, we examined and compared several types of these electrodes (e.g., Co HCF-activated polycrystalline graphite; $\mathrm{Co}$, Ni and $\mathrm{Cu} \mathrm{HCF}$-activated carbon felts; Ni HCF-activated metallic nickel). It was shown that, when these electrodes are used in conjunction with EC-induced cycles of iron reduction and oxidation, the cesium can be transferred from high-ionic strength solutions with low Cs concentrations to a much smaller volume of a low-ionic strength solution (regenerant). The concentration of Cs in the regenerant can exceed that in the waste by several orders of magnitude. Therefore, the goal of separating and concentrating radioactive cesium from a much more complex matrix can be achieved using EIX. The performance of EIX is affected by both the solution composition (notably, its $\mathrm{pH}$ ) and the properties of the electrode (e.g., its porosity, mass transfer to and 
from the HCF phase and its intrinsic selectivity). Although the project demonstrated the potential to use EIX technology to collect cesium from the Hanford tank wastes, more work needs to done to improve the EIX technology, particularly the stability of the electrodes.

The research conducted as part of the project has both direct, immediate applicability to an important waste treatment need of DOE, and it also expands the knowledge base regarding adsorption of metals and EIX processes in general. This study bridged several scientific areas and yielded valuable knowledge for implementing new technological processes. The applicability of the results extends beyond the highly specialized application niches investigated experimentally to other issues of potential interest for EMSP programs (e.g., separation of chromium from a variety of wastes using IOCS, separation of Cs from neutral and acidic wastes with EC-controlled HCFs).

Due to the high selectivity of IOCS for Sr, its chemical stability, low cost, ease of preparation, and the absence of any apparent problems related to its long term storage or vitrification, the use of this material appears to be a highly promising option for Sr removal from high level liquid tank wastes. In our opinion, this material is ready for use in pilot-scale experiments. We believe that its employment can significantly accelerate the pace and reduce the costs of the on-site treatment of the tank wastes.

The use of EC-controlled HCFs for Cs separation has great practical promise, but its application requires more work to improve its chemical stability, notably at high $\mathrm{pHs}$. It is also necessary to develop electrodes that overcome limitations associated with mass transfer and capture of the regenerant by the pores in the active material; however, these challenges do not appear to be particularly severe. In the long term, we envision the use of HCF-based electrochemical systems to control Cs in a variety of aqueous media (not necessarily having high $\mathrm{pH})$. Since EC control enables regeneration of the hexacyanoferrate exchanger, much 
benefit is expected due to the significant reduction or complete elimination of any long-term storage of the HCFs.

Three Master's theses were completed as part of this project. The titles and authors of those theses are, "Separation of strontium from low-organic simulated hanford tank waste by adsorption onto metal oxides," by Birgitte O. Hansen (1998), "The effects of chelators on strontium adsorption onto iron oxide- coated sand", by Pierre Kwan (1999), and "Separation of cesium from radioactive wastes using hexacyanoferrates and electrochemically switched ion exchange", by Mark D. Jensen (2000). The theses are available through the University of Washington library system. In addition to that, a major part of a Ph.D. thesis was carried out as part of this project. The title and author of this work are "Preparation, characterization and sorption properties of $\mathrm{Ni}(\mathrm{II})$ in single and combined solid phases and separation of $\mathrm{Cs}^{+}$from simulated Hanford waste using electrochemically controlled ion exchange" by Henrik GreenPetersen (1999). Although this work was done predominantly at University of Washington, the thesis was defended at Aarhus University in Denmark and is available from that university. 


\section{Research Objectives}

The objectives of this project were to develop new technologies that might be applicable to treatment of $\mathrm{Sr}$ and $\mathrm{Cs}$ in the extremely complex tank wastes stored at the Hanford DOE site, to test those technologies at bench-scale using simulated waste solutions, and to expand the scientific and engineering understanding of the principles underlying the processes. The specific processes to be tested for $\mathrm{Sr}$ treatment were adsorption of $\mathrm{Sr}$ onto iron and titanium oxides prepared in various ways, and combinations of $\mathrm{Sr}$ adsorption with either microfiltration or regeneration of the adsorbent. The process to be tested for Cs removal was electrochemically controlled ion exchange using hexacyanoferrates as the ion exchange medium, embedded in or otherwise associated with an electrode whose electrical potential could be independently controlled.

The relevance of the project of the project is simple and direct: removal of Sr and Cs from the tank wastes is a problem that is both urgent and long-standing, and to which numerous potential treatment processes have been applied. The processes studied were related to those that have been employed in prior research, but they differed in substantial ways that made them potentially more effective and less expensive.

\section{Methods}

Sr removal from solution and retention by several inorganic adsorbents was studied in batch and column experiments using a synthetic supernatant (SS) that represented the typical composition of single-shell Hanford tanks. ${ }^{1,2} \mathrm{SS}$ contained up to $5.5 \mathrm{M} \mathrm{Na}^{+}, 3.70 \mathrm{M} \mathrm{NO}_{3}{ }^{-}$, $1.24 \mathrm{M} \mathrm{OH}^{-}, 0.6 \mathrm{M} \mathrm{CO}_{3}{ }^{2-}, 0.23 \mathrm{M} \mathrm{Al}^{3+}, 0.05 \mathrm{M} \mathrm{SO}_{4}{ }^{2-}$ and $0.015 \mathrm{M} \mathrm{F}^{-}$. Minor cationic components of SS included potassium, calcium and chromium at concentrations 0.08 , $1.1 \times 10^{-4}$ and $7.7 \times 10^{-5} \mathrm{M}$, respectively. The concentration of $\mathrm{Sr}$ in SS varied from 1.14 to $3.42 \mu \mathrm{M}$ (100 to $300 \mu \mathrm{g} / \mathrm{L}$ ). Up to $2 \times 10^{-4} \mathrm{M}$ EDTA was added to SS to investigate $\mathrm{Sr}$ removal from solutions containing organic chelating agents. In some cases, batch experiments were combined with filtration using hollow-fiber or flat-sheet membranes. Adsorbents tested in the batch/filtration experiments for $\mathrm{Sr}$ removal included amorphous iron oxide (doped, in some cases, with silicate), goethite, hematite, rutile, and anatase.

Column adsorption studies used only iron-oxide-coated sand (IOCS) as the adsorbent, prepared according to Bailey (1992). Standard sand was first soaked in sulfuric acid, rinsed and dried. The coating solution was made by dissolving iron nitrate in water and adjusting its $\mathrm{pH}$ with sodium hydroxide. The cleaned sand was covered with the coating solution and baked at $110^{\circ} \mathrm{C}$ for 14 hours. The product was sieved to yield the 0.59 to $1.18 \mathrm{~mm}$ fraction, which was heated at $110^{\circ} \mathrm{C}$ for an additional three hours. Bennett (1992) reported that IOCS prepared in this way has a packed dry density of $1.66 \mathrm{~g} / \mathrm{mL}$, an iron concentration of $2.1 \%$ by weight, and a surface area of $2.40 \mathrm{~m}^{2} / \mathrm{g}$.

\footnotetext{
${ }^{1}$ Chaiko, D.J., Y.Vojta, M.Takeuchi (1995). Extraction of technetium from simulated Hanford tank wastes. Separation Sci. Technol., 30, 1123-1137.

${ }^{2}$ Bell, J.T., L.H.Bell (1995). Separation technology: the key to radioactive waste minimization. In Chemical Pretreatment of Nuclear Waste for Disposal, edited by W.W.Schulz and E.P.Horwitz, Plenum Press, NY, pp. 135 .
} 
SS was continuously fed into a series of three IOCS-packed columns, using an empty bed contact time (EBCT) of 5 min in each bed. Every three days the upstream column was taken off-line, regenerated, and placed back into the treatment train in the most downstream position. Two bed volumes of deionized (DI) water were passed downflow through the column before it was regenerated. Then $100 \mathrm{~mL}$ of water acidified with $\mathrm{HNO}_{3}$ to $\mathrm{pH} 2.5$ to 3 was recirculated through the column for one hour at a flow rate of $30 \mathrm{~mL} / \mathrm{min}(20$ seconds EBCT). If needed, $\mathrm{HNO}_{3}$ was added to the regenerant to maintain the target $\mathrm{pH}$. The concentrations of target elements (e.g., $\mathrm{Sr}, \mathrm{Ca}, \mathrm{Cr}$ ) in the regenerant were determined after each regeneration. Occasionally, precipitates accumulated within the columns. In such cases, the columns were backwashed prior to the regeneration, using a flow rate just high enough to fluidize the bed.

Cesium removal was investigated from high-ionic strength solutions (up to $10 \mathrm{M}$ ). None of the adsorbents used in Sr treatability tests retained cesium, so the experiments addressing cesium removal used hexacyanoferrates (HCFs) as the active media for collecting Cs. In these experiments, the oxidation state of the HCFs was controlled by an electrochemical (EC) system consisting of a three-electrode cell and a computer-controlled potentiostat. Several materials were tested as electrode substrates, including polycrystalline graphite rods, carbon felt, and nickel wire and gauze. Counter electrodes were made of polycrystalline graphite sheet or platinum wire. Standard calomel or $\mathrm{Ag} / \mathrm{AgCl}$ electrodes were used as reference electrodes. HCFs of $\mathrm{Ni}$, Co or $\mathrm{Cu}$ were incorporated into the graphite electrodes either by repetitive soaking of the electrodes in solutions containing potassium hexacyanoferrate $\left(\mathrm{K}_{4} \mathrm{Fe}(\mathrm{CN})_{6}\right)$ and the nitrate of the respective metal, or by EC deposition of the HCF phase. Only EC deposition was used for Ni electrodes.

The metal HCF electrodes were loaded with $\mathrm{Cs}^{+}$from the influent at $0 \mathrm{~V}$ and unloaded in a solution containing potassium nitrate (the regenerant) at $\mathrm{E}=+1.0 \mathrm{~V}$. Following the unloading, the electrode was transferred to the influent, and the loading/unloading cycle was repeated. The concentrations of potassium and cesium in the effluent ranged from $10^{-3}$ to $10^{-1} \mathrm{M}$ and from $10^{-6}$ to $10^{-2} \mathrm{M}$, respectively. The treatment technique for Cs is referred to below as 'electrochemical ion exchange', or EIX. The concentration of Cs in the influent and regenerant were analyzed by flame atomic absorption spectrophotometry (AAS), and the concentrations of all other metallic species of interest were determined using a Jobin- Yvon Ultratrace-138 inductively coupled plasma spectrometer.

\section{Results}

Batch experiments demonstrated that the different adsorbents investigated had distinctly different abilities to retain $\mathrm{Sr}$. Amorphous iron oxide had the highest adsorption capacity and retained Sr over the broadest $\mathrm{pH}$ range. Regeneration of amorphous iron oxide was easily achieved at $\mathrm{pH} \sim 5$. None of the adsorbents was able to retain cesium.

Attempts were made to combine $\mathrm{Sr}$ retention by of iron oxides with membrane microfiltration of the SS. However, when amorphous iron oxide was used as the adsorbent, both hollow-fiber membranes and flat-sheet membranes used in stirred-cell systems fouled rapidly and irreversibly. Hematite did not foul the membranes, but it removed only $\sim 50 \%$ of the Sr under the conditions tested, and the hematite was incompletely regenerated (i.e., some Sr was not released from the adsorbent) when the suspension was acidified. In addition, hematite was less selective $\mathrm{Sr}$ than amorphous iron oxide was, retaining calcium and aluminum along with the target contaminant. Preliminary tests of Sr retention by IOCS 
suggested that that material was selective for $\mathrm{Sr}$, could be easily regenerated, and that it had almost as large a capacity for $\mathrm{Sr}$ as does amorphous iron oxide. Since the physical properties of freely suspended amorphous iron oxide complicate its separation from solution, subsequent work focused on the use of IOCS as the adsorbent of choice.

When SS without organic complexing agents was used as influent, the $\mathrm{Sr}$ concentration exiting the final column was below the detection limit $(\sim 1 \mu \mathrm{g} / \mathrm{L})$ during the first 24,000 bed volumes treated (Figure 1) (note: number of bed volumes is based on flow through a single column). Thereafter, a relatively short-term failure of the $\mathrm{pH}$ probe led to overloading of the adsorbent and breakthrough of some $\mathrm{Sr}$ into the effluent. After the problem was corrected, $\mathrm{Sr}$ breakthrough decreased markedly. Once $>30,000$ bed volumes of influent had been treated, low levels of $\mathrm{Sr}$ reappeared in the effluent. In total, the system treated 50,562 BV of the influent without interruption over a 30-week span, achieving a cumulative $\mathrm{Sr}$ retention efficiency of $94 \%$ (Figure 2); the overall retention efficiency excluding the period when the $\mathrm{pH}$ probe malfunctioned was $97 \%$. Prior to that malfunction, the retention efficiency was practically $100 \%$.

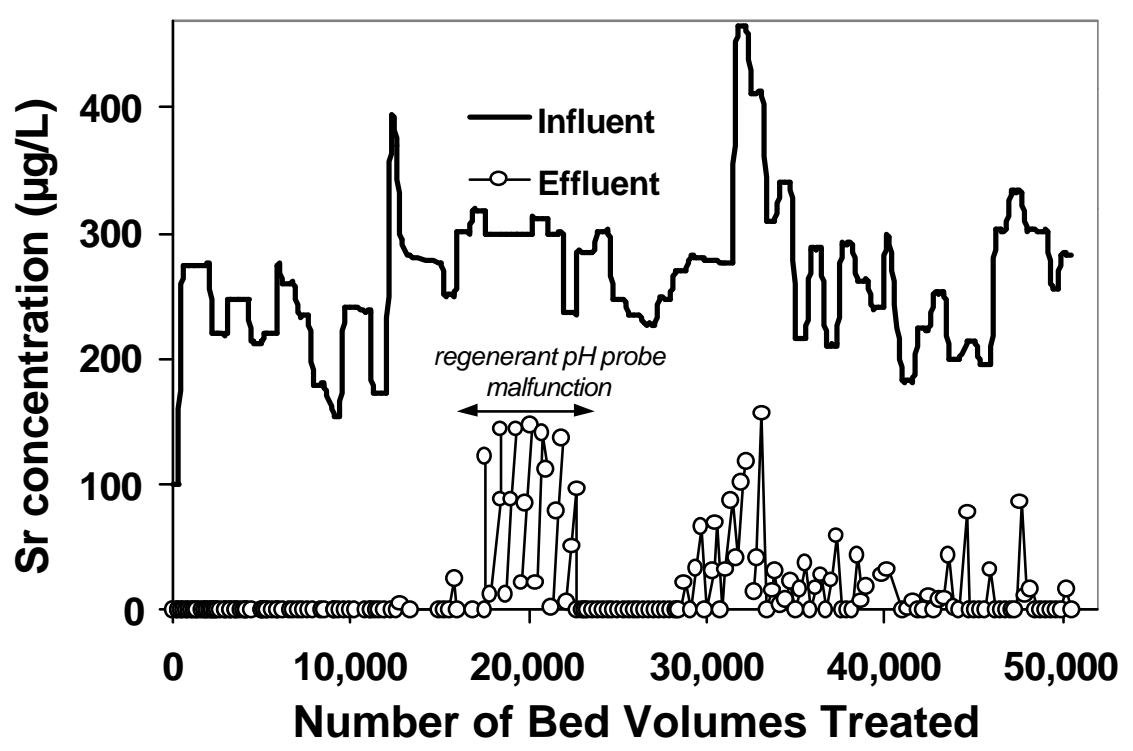

Figure 1. Strontium concentrations in the system containing three IOCS-packed columns. Feed was synthetic supernatant without EDTA. 


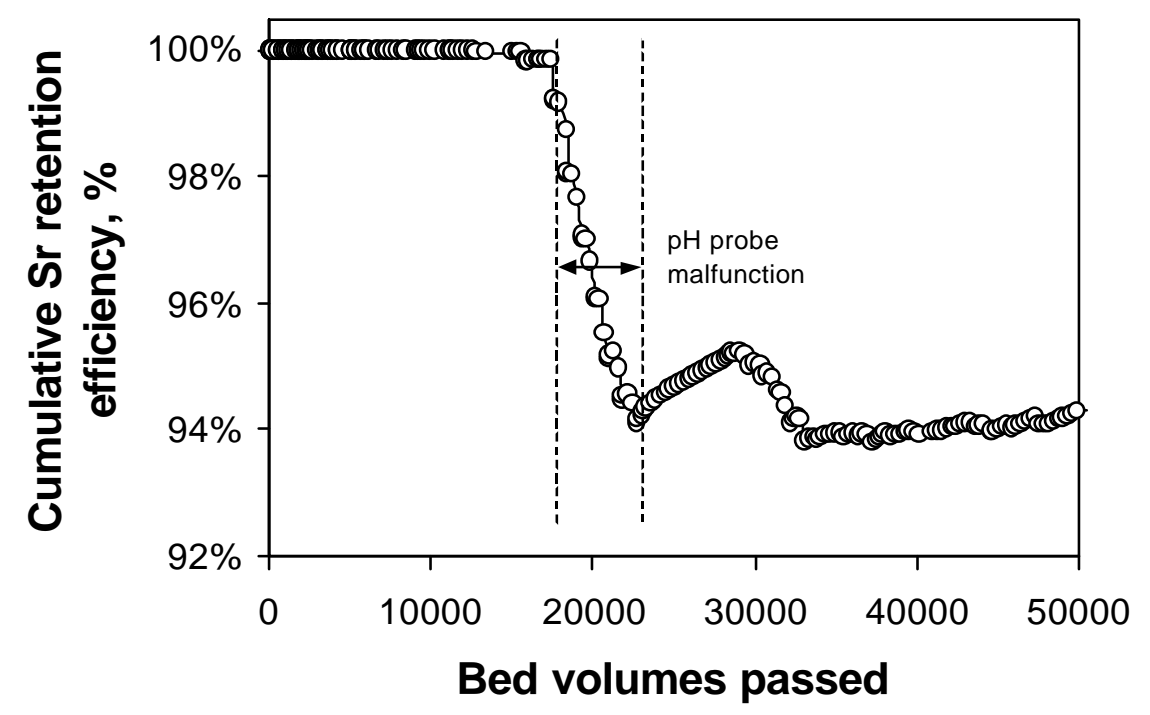

Figure 2. Cumulative strontium retention efficiency by three IOCS-packed columns; same conditions as Figure 1.

Although the IOCS could be regenerated at $\mathrm{pH} 5$, the speed and efficiency of regeneration were better at lower $\mathrm{pH}$, so regeneration was routinely conducted at $\mathrm{pH}$ between 2.5 to 3.0. Good regeneration efficiencies were obtained over 64 treatment/ regeneration cycles (16 on each column) (Figure 3). The regenerant solution was re-used during this portion of the test period, allowing an overall waste volume reduction of $>150$-fold.

A small percentage of the retained $\mathrm{Sr}$ was not released during the regeneration process and was apparently incorporated into the adsorbing medium. When the IOCS was subjected to acid digestion at the end of the experiment, the mass of $\mathrm{Sr}$ released corresponded to approximately $76 \%$ of the difference between the cumulative amount retained during all the previous tests and the cumulative amount recovered in all the regeneration steps. As is clear from Figure 1, the accumulation of unrecoverable $\mathrm{Sr}$ in the adsorbent had no discernable effect on subsequent $\mathrm{Sr}$ adsorption. 


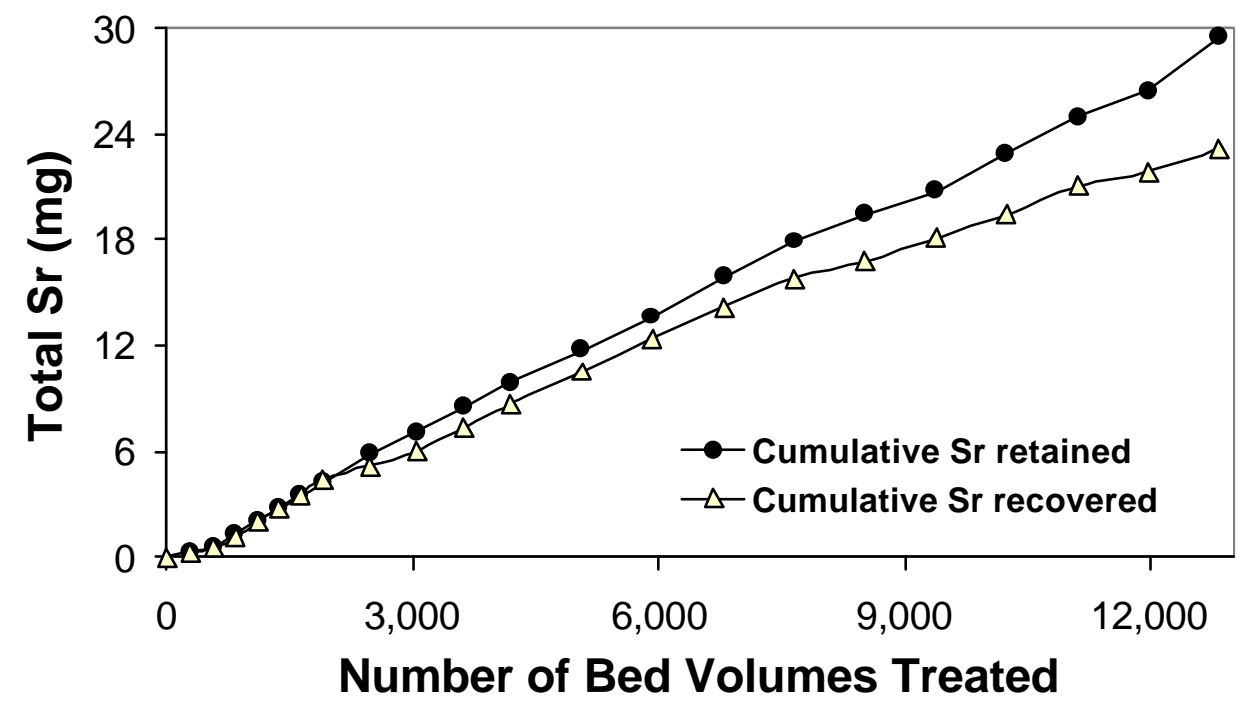

Figure 3. Cumulative Sr retained from SS and recovered by regeneration. Test run for $2,000 \mathrm{BV}$ to 13,000 bed volumes.

The IOCS did not retain $\mathrm{Al}$ and $\mathrm{Cr}$ from the $\mathrm{SS}$, and the amount of $\mathrm{Ca}$ that adsorbed was much less than that of Sr. The vast majority of the adsorbed Ca was released in the regeneration step. No dissolution of iron from the IOCS was detected over the course of the experiments. In fact, the concentration of iron in the influent was consistently higher than in the effluent. Thus, the IOCS was highly selective for $\mathrm{Sr}$, and the iron oxide coating was very stable under the test conditions.

The presence of EDTA dramatically reduced Sr retention by the IOCS. In these tests, the $\mathrm{Sr}$ concentration in the effluent depended not only on the EDTA concentration, but also on the calcium concentration, reflecting competition between $\mathrm{Ca}$ and $\mathrm{Sr}$ for the organic ligand.

Specifically, when the concentration of EDTA was less than that of calcium, Sr retention by the IOCS was relatively efficient. For instance, $\mathrm{Sr}$ was retained with almost $90 \%$ efficiency during treatment of 13,000 bed volumes of SS when the influent EDTA/Ca molar ratio was 0.75 (Figure 4). However, increasing the EDTA concentration to values greater than the stoichiometric requirement for binding $\mathrm{Ca}$ led to an immediate and dramatic increase in the $\mathrm{Sr}$ concentration in the effluent. The concentrations of $\mathrm{Ca}$ and $\mathrm{Cr}$ in the effluent increased along with that of Sr when the EDTA concentration was increased (Figure 5 and Figure 6). This result suggests that controlling the calcium concentration in nuclear waste streams that contain organic complexing agents may be critical for assuring $\mathrm{Sr}$ retention. Adding $\mathrm{Ca}$ as needed to facilitate $\mathrm{Sr}$ retention appears to be a practical option, since $\mathrm{Ca}$ is not toxic and is not known to cause problems in vitrification processes. 


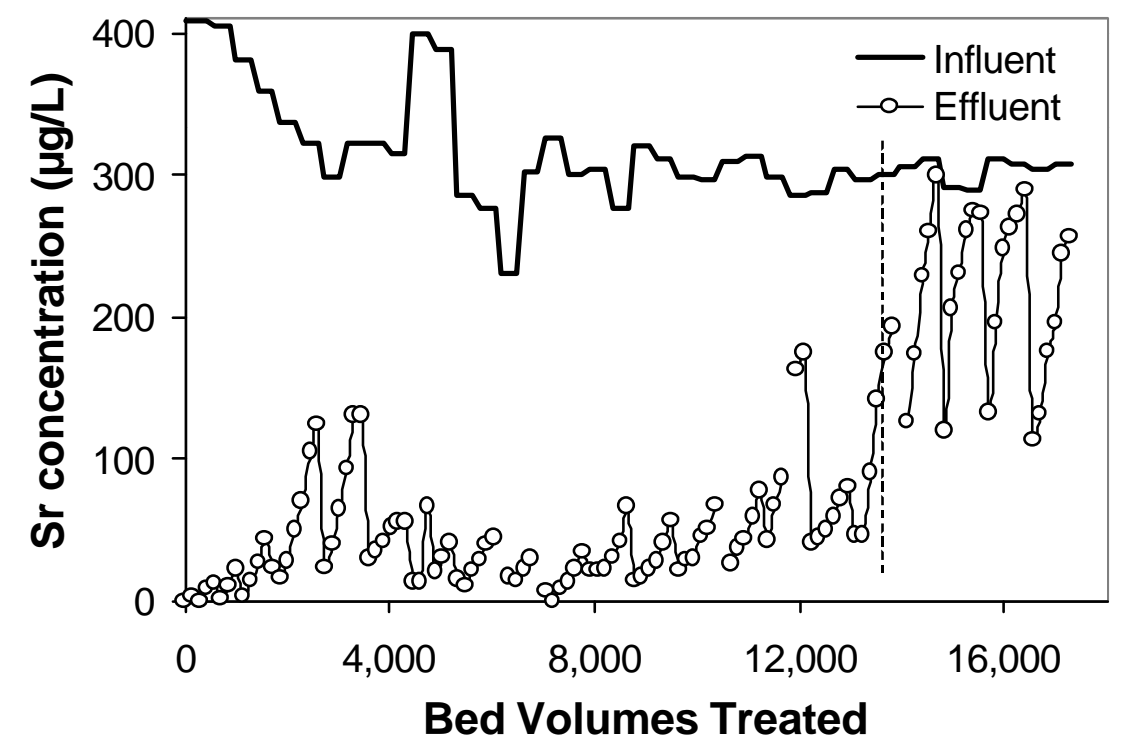

Figure 4. Influent and effluent Sr concentrations from the system of the IOCS-packed columns. Carbonate-free synthe tic supernatant. [EDTA]/[Ca] molar ratio 0.75 . The dashed line indicates increase of EDTA concentration to a [EDTA]/[Ca] ratio of 1.50.

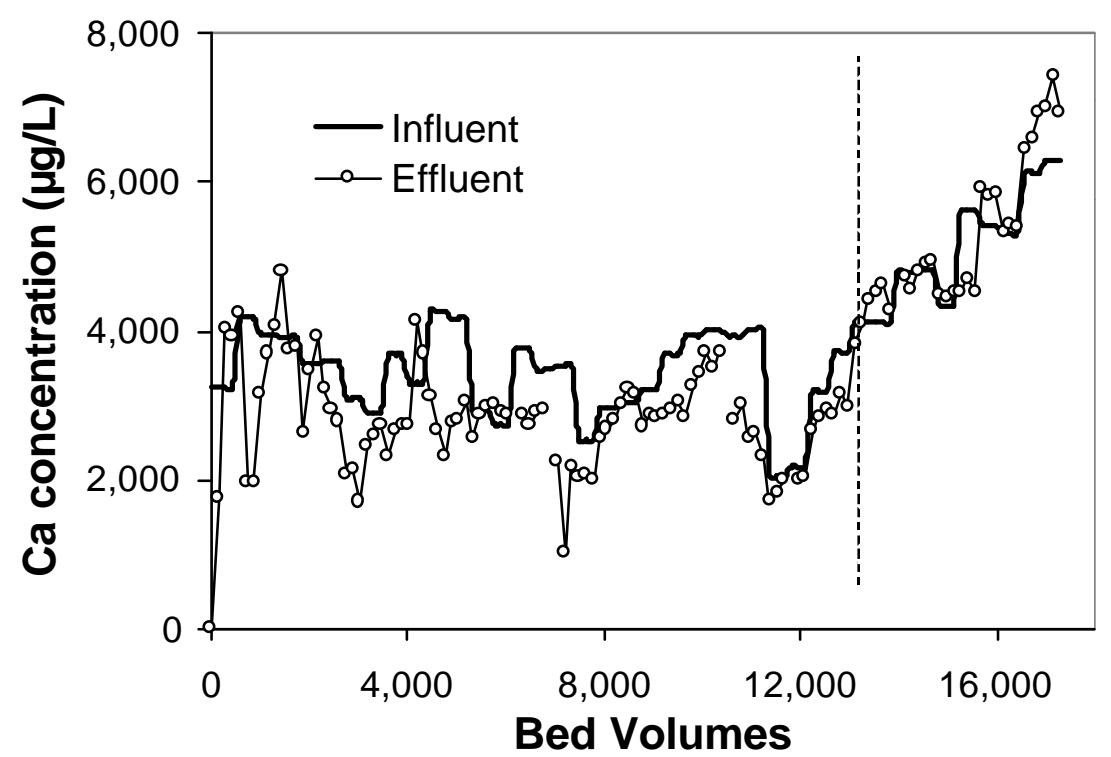

Figure 5. Influent and effluent Ca concentrations for the system of the IOCS-packed columns. Carbonate-free synthetic supernatant. [EDTA]/[Ca] molar ratio 0.75. The dashed line indicates the increase of EDTA concentration to a [EDTA]/[Ca] ratio of 1.50. 


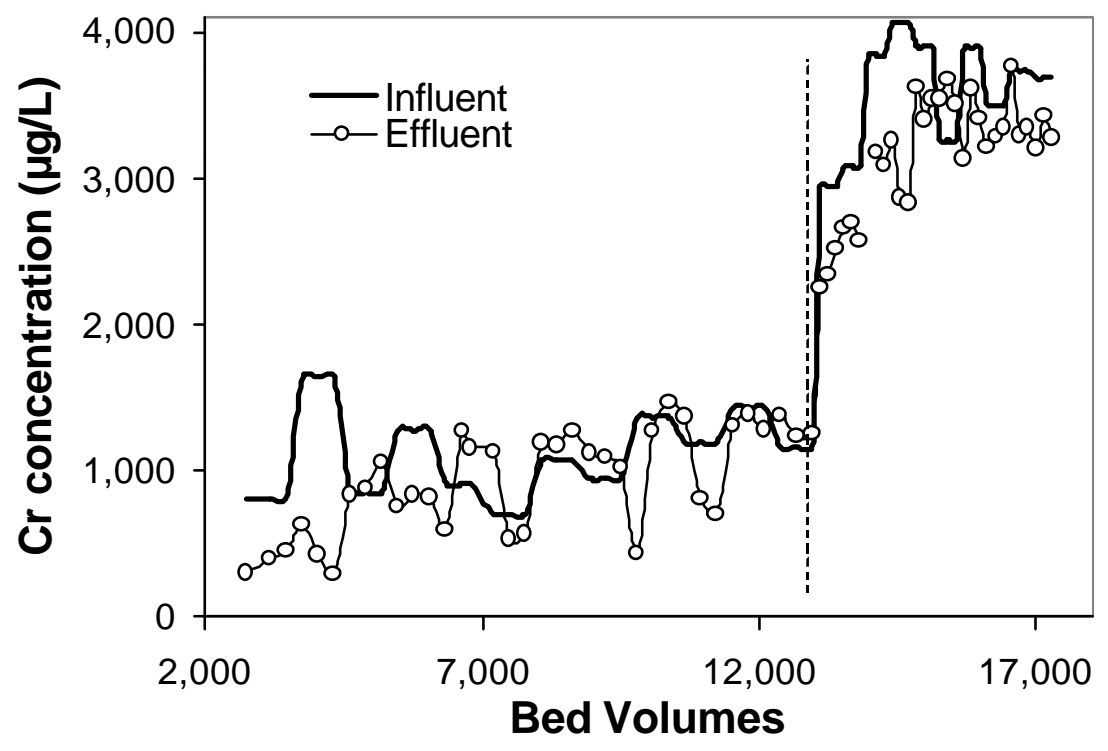

Figure 6. Influent and effluent chromium concentrations for the system of the IOCSpacked columns. Carbonate-free synthetic supernatant. [EDTA]/[Ca] molar ratio 0.75. The dashed line indicates the increase of EDTA concentration to a [EDTA]/[Ca] ratio of 1.50.

$\mathrm{Sr}$ adsorption on IOCS simultaneously concentrates $\mathrm{Sr}$ and isolates it from other contaminants, transferring it from a large volume of a very complex waste stream to a small volume of regenerant containing $\mathrm{Sr}$ as the dominant contaminant. If desired, the regenerant could be evaporated or otherwise treated to yield a trivially small amount of material ready for ultimate disposal. Potential advantages of the use of IOCS over existing technologies for Sr removal result from the fact that IOCS is a low-cost highly selective and easily reusable/regenerable material. No new material need be added to the waste before or during treatment, no new sludge is generated, and no new problems with solid/liquid separation are created. The incremental operating costs for both treatment and ultimate disposal are small.

As indicated in the Methods section, cesium removal from SS was investigated using electrochemical ion exchange, or EIX, with hexacyanoferrates (HCFs) as the active ion exchange media. For cobalt HCF, which was one of the materials that showed the greatest promise for Cs removal, an idealized chemical formula of the active phase is $\mathrm{Me}_{n}\left[\mathrm{CoFe}(\mathrm{CN})_{6}\right]$, where Me is a cation reversibly exchangeable for cesium, and the value of $\mathrm{n}$ is 1 or 2. The oxidation state of the iron in the solid is electrochemically controlled by an external potentiostat. Theoretically, the amount of cesium that can be retained by the electrode is established by the amount of the active phase present and the oxidation state of the hexacyanoferrate iron in that phase.

The reduction of $\mathrm{Fe}(\mathrm{III})$ to $\mathrm{Fe}(\mathrm{II})$ perturbs the charge balance of the HCF matrix and, if the electrode is immersed in a cesium-containing solution, initiates the influx of cesium ions to restore the electroneutrality. Subsequent oxidation of $\mathrm{Fe}(\mathrm{II})$ to $\mathrm{Fe}$ (III) in a regenerating solution causes the cesium to be expelled from the HCF matrix, restoring the electrode capacity for cesium. In theory, these cycles of Fe reduction combined with Cs retention, followed by Fe oxidation combined with Cs release, can be repeated indefinitely. The overall reaction sequence is summarized in Figure 7. Note that the HCF phase is not used to store the 
radioactive Cs, but is employed only to transfer the Cs from the influent to the regenerant. Therefore, problems associated with long-term storage of Cs-laden HCF potentially are minimized or eliminated.

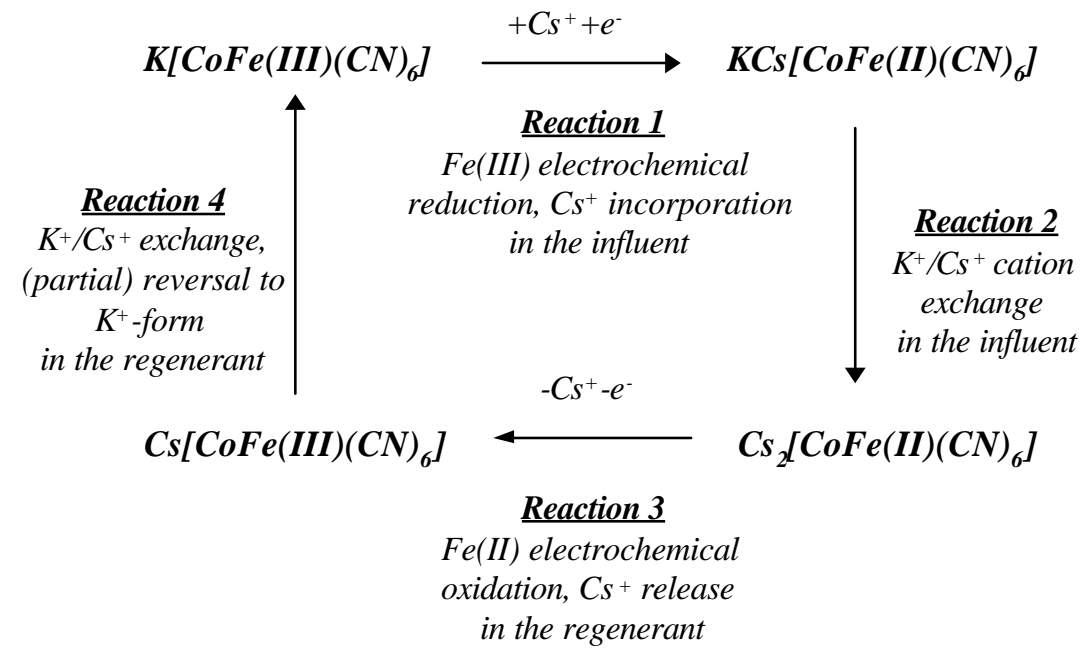

Figure 7. Idealized sequence of electrochemical and ion-exchange reactions that affect the transfer of Cs during EIX treatment.

Experimental studies focused on the feasibility of carrying out the cyclic process described above. Three types of experiments were conducted. First, ion exchange reactions and stability of several non-electrochemically controlled cesium-active $\mathrm{HCFs}$ ( $\mathrm{Fe}, \mathrm{Co}, \mathrm{Cu}$, $\mathrm{Zn}, \mathrm{Ni}, \mathrm{Cd}$ ) were examined at varying $\mathrm{pHs}$, ionic strengths, reaction times. Cobalt $\mathrm{HCF}$ was found to be superior to the others in terms of $\mathrm{pH}$ stability and selectivity to cesium. Consistent with previous literature reports, however, the HCFs were not stable (i.e., they decomposed, most likely forming soluble $\left[\mathrm{Fe}(\mathrm{CN})_{6}\right]^{4-}$ and the hydroxide of the respective heavy metal) at $\mathrm{pH}$ values higher than 11 to 12 . The cost and complexity of lowering the $\mathrm{pH}$ of the intensely basic Hanford wastes to values where the HCFs are stable appears to be a major limitation of the applicability of this process in such systems.

In a second phase of experimental work, the electrochemistry of hexacyanoferratemodified graphite and carbon cloth electrodes was studied. Activation of the electrodes with $\mathrm{HCF}$ dramatically increased the current through them. The performance of the HCF electrodes deteriorated after repetitive oxidation/reduction cycles (Figure 8), as has been reported in the literature previously. ${ }^{3,4}$. However, it was possible to circumvent this limitation by subjecting the electrode to EC cycling in a solution containing the reagent needed to deposit the HCF on the electrode surface (Figure 9).

\footnotetext{
${ }^{3}$ Lilga, M.A., R.J.Orth, J.P.H.Sukamto, S.M.Haight, D.T.Schwartz. (1997). Metal ion separation using electrochemically switched ion exchange. Separation Purification Technol., 11, 147-158.

${ }^{4}$ Sinha, S., B.D.Humphrey, A.B.Bocarsly (1984). Reaction of nickel electrode surfaces with anionic metal cyanide complexes: formation of surface precipitates. Inorg. Chem., 23: 203-212.
} 


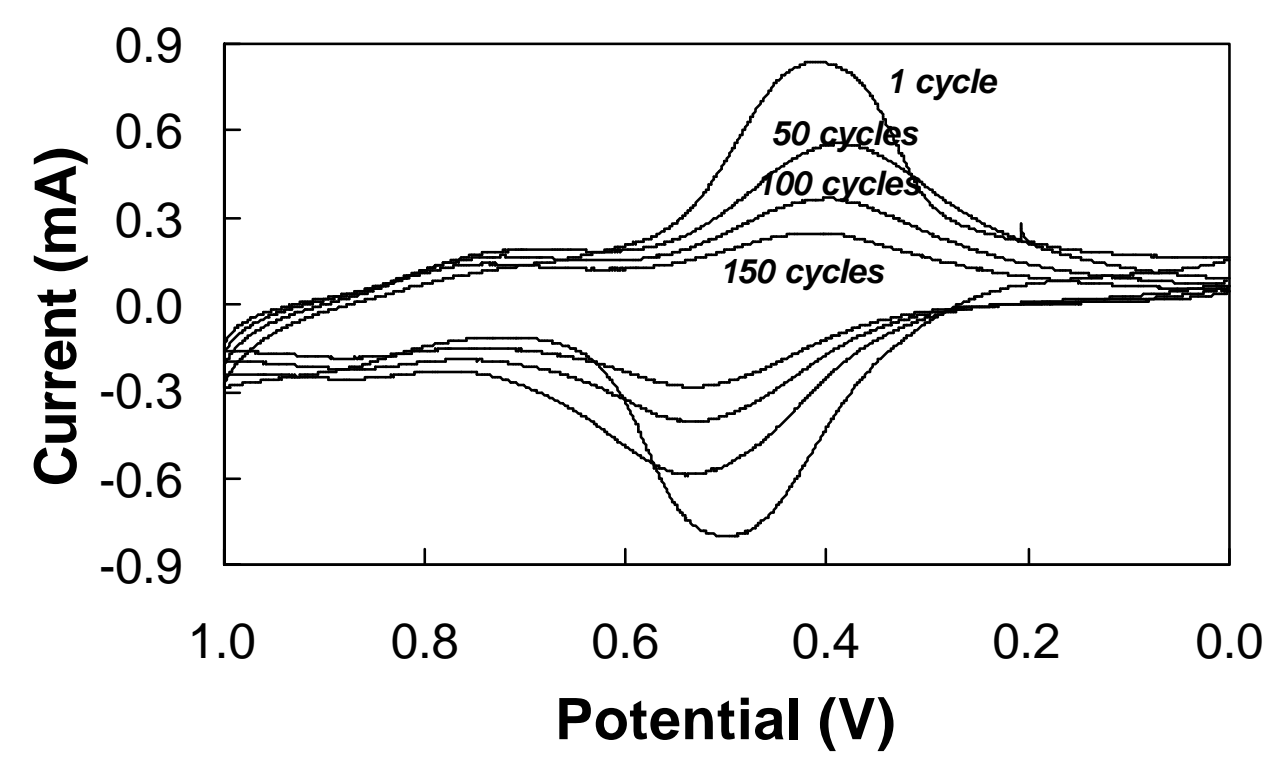

Figure 8. Degradation of Co HCF pha se deposited onto carbon felt associated with repetitive reduction/oxidation cycles. Cyclic voltammograms taken in $1.0 \mathrm{M} \mathrm{NaNO}_{3}$ at a scan rate of $100 \mathrm{mV} / \mathrm{s}$.

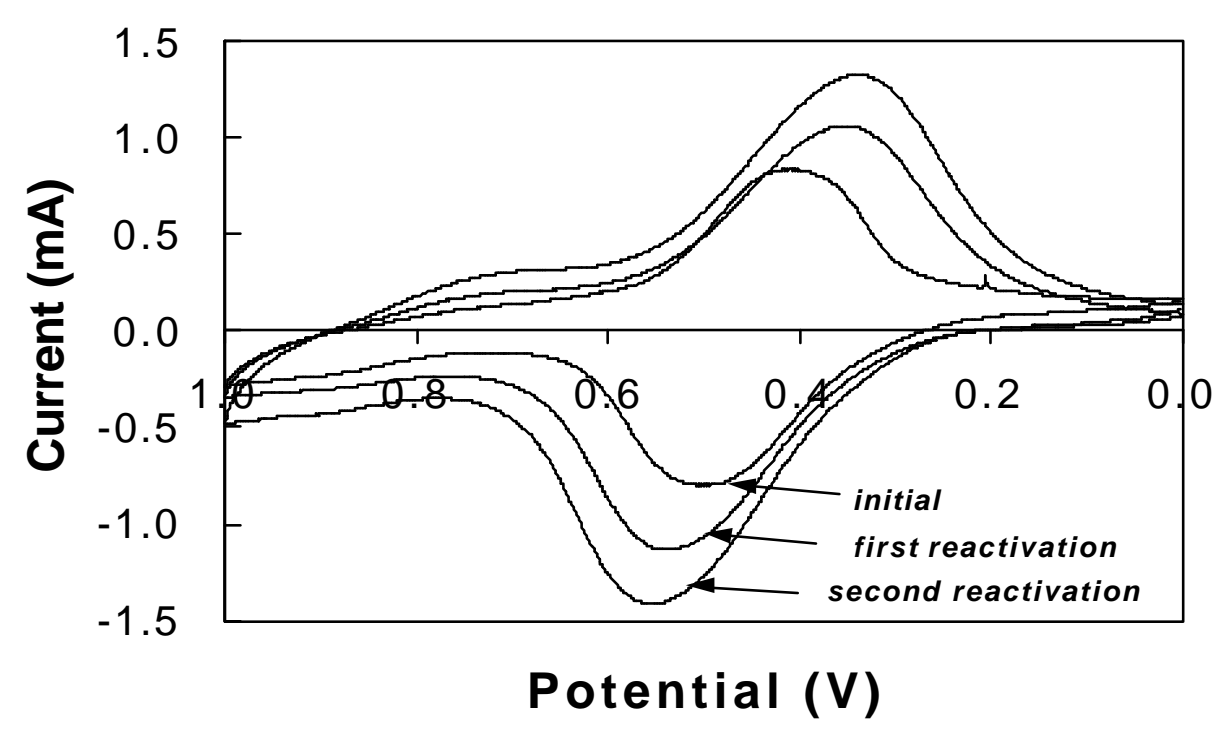

Figure 9. Reactivation of Co HCF active phase electrochemically deposited on carbon felt. Initial Co-HCF was deposited onto carbon felt by 15 oxidation/reduction cycles between 0 and $1.0 \mathrm{~V}$, at a scan rate of $100 \mathrm{mV} / \mathrm{s}$ in $5 \times 10^{-4} \mathrm{M} \mathrm{K}_{3} \mathrm{Fe}(\mathrm{CN})_{6}, 5 \times 10^{-4} \mathrm{M} \mathrm{Co}\left(\mathrm{NO}_{3}\right)_{2}$, and 1.0 $\mathrm{M} \mathrm{NaNO}_{3}$. Each reactivation step consisted of five such oxidation/reduction cycles. Each reactivation cycle was followed by 100 treatment cycles. Cyclic voltammograms were recorded under the same conditions as in Figure 8.

X-ray photoelectron spectroscopy confirmed that the Cs incorporated in the HCF phase largely disappeared following the oxidation of the Fe in the HCF (Figure 10). The presence of 
potassium in the regenerant was important for achieving quantitative Cs release, indicating that both favorable cation- exchange equilibria and electrochemical control are important for achieving the desired result. The amount of cesium taken up by the electrode was qualitatively consistent with the change in charge associated with the oxidation/reduction of the Fe in the HCF. For Co HCF graphite rod electrodes, the amount of Cs transferred to the regenerant depended on the concentration of Cs in the influent and corresponded to $\sim 40 \%$ of the charge transferred in the iron oxidation/reduction reaction (Figure 11). Sodium concentrations of up to $5 \mathrm{M}$ did not interfere with the performance of the electrode.

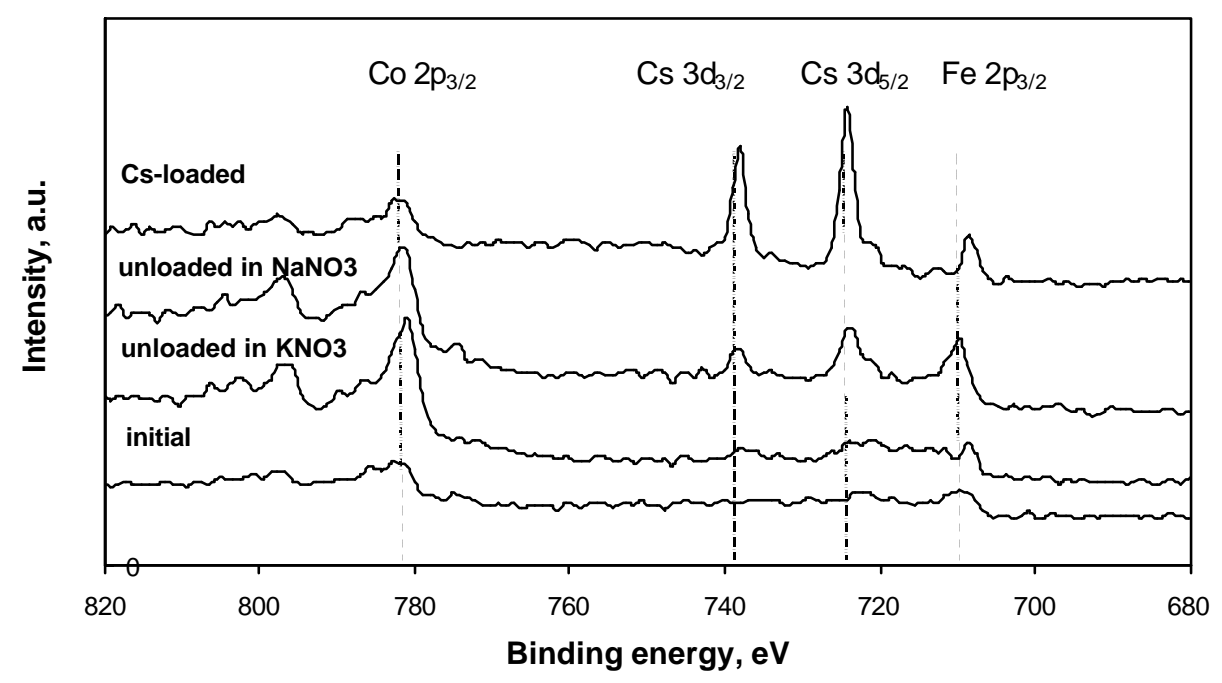

Figure 10. Comparison of X-ray photoelectron spectra of Co HCF electrodes prior to loading with Cs, loaded in the influent, and unloaded in sodium or potassium nitrate regenerants.

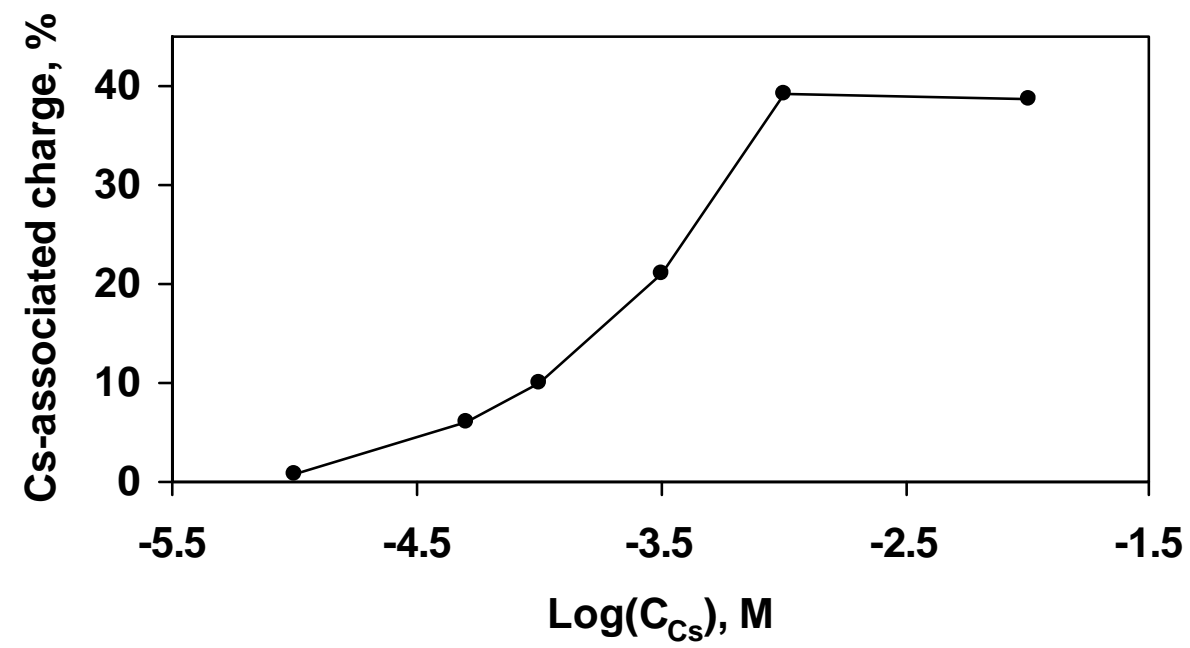

Figure 11. Correlation between the concentration of $\mathrm{Cs}^{+}$in the influent and the $\mathrm{Cs}^{+}-$ associated charge. Co HCF graphite rod electrode. The cesium was loaded at $0 \mathrm{~V}$ in $1 \mathrm{M}$ $\mathrm{NaNO}_{3}$ from solutions with varying Cs concentrations and was unloaded at $+1.0 \mathrm{~V}$ in $0.5 \mathrm{M}$ $\mathrm{KNO}_{3}$. 
Further experiments demonstrated that, while the graphite and carbon felt-based HCF electrodes had substantial capacity for binding $\mathrm{Cs}$ and could be easily reactivated, the porosity of these materials was problematic in multi cycle Cs transfer experiments.

Specifically, regenerant solution with a high Cs concentration was trapped in the pores of the electrode and was being carried over to the influent. Although this does not constitute a fundamental flaw in the concept of EIX, better design of the electrodes and/or a more elaborate routine to reduce carryover of fluid from the regenerant to the influent (e.g., rinsing prior to exposure to the influent) needs to be developed.

To circumvent this problem in the lab-scale system, the next set of experiments used smooth Ni electrodes, which were electrochemically activated with HCF. (Attempts to deposit $\mathrm{Co} \mathrm{HCF}$ on metallic cobalt failed, because the Co HCF layer did not adhere well to the surface; by contrast, a Ni HCF layer adhered tenaciously to nickel metal). As shown in Figure 12 and Figure 13, it was possible to transfer Cs from the influent to a regenerant solution containing a much higher concentration of this cation using this setup. If the regenerant contained little or no cesium, some Cs could be transferred without electrochemical control; however, no transfer occurred without the assistance of EC control when the regenerant contained a high Cs concentration.

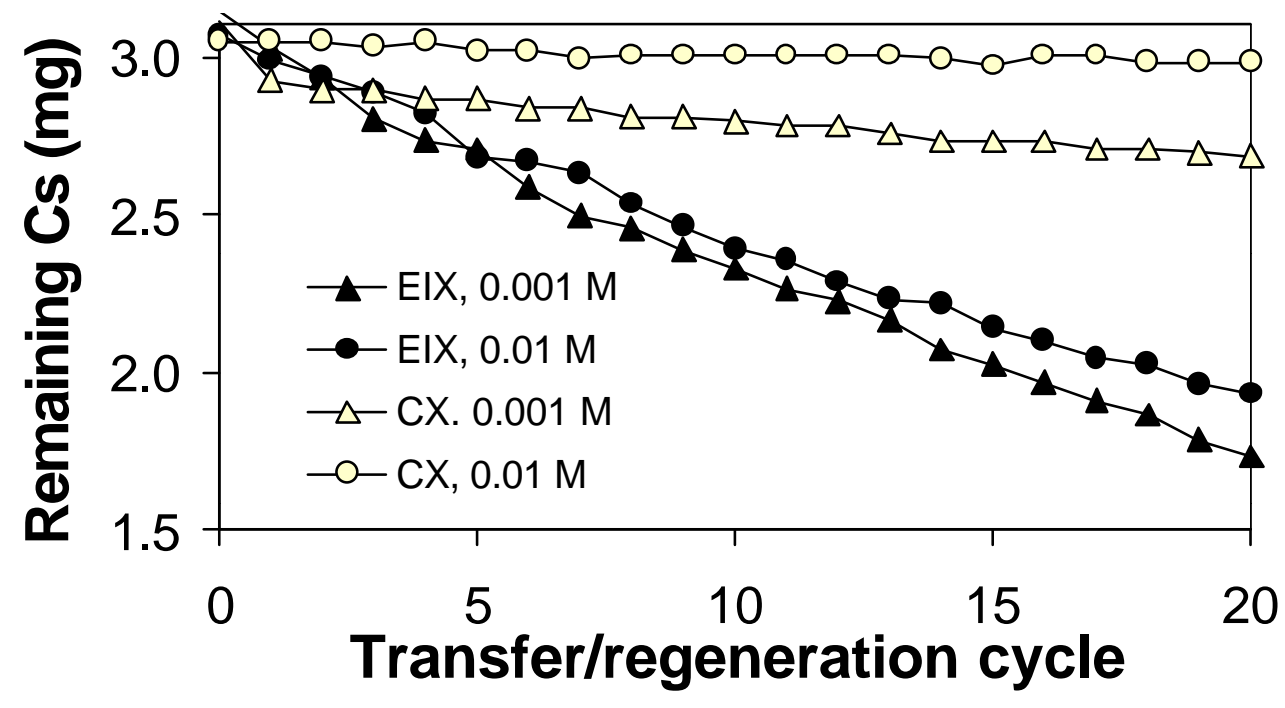

Figure 12. Comparison of the performance of cation exchange (CX) and EIX modes for Cs transfer from solutions containing $0.0001 \mathrm{M}$ Cs to regenerant solutions containing higher Cs concentrations. HCF-activated Ni mesh electrode. HCF was deposited on the Ni-mesh by polarizing the electrode at $1.2 \mathrm{~V}$ for 35 minutes in $1.0 \mathrm{M} \mathrm{NaNO}_{3}$ and $2.0 \mathrm{mM} \mathrm{K}_{3} \mathrm{Fe}(\mathrm{CN})_{6}$. 


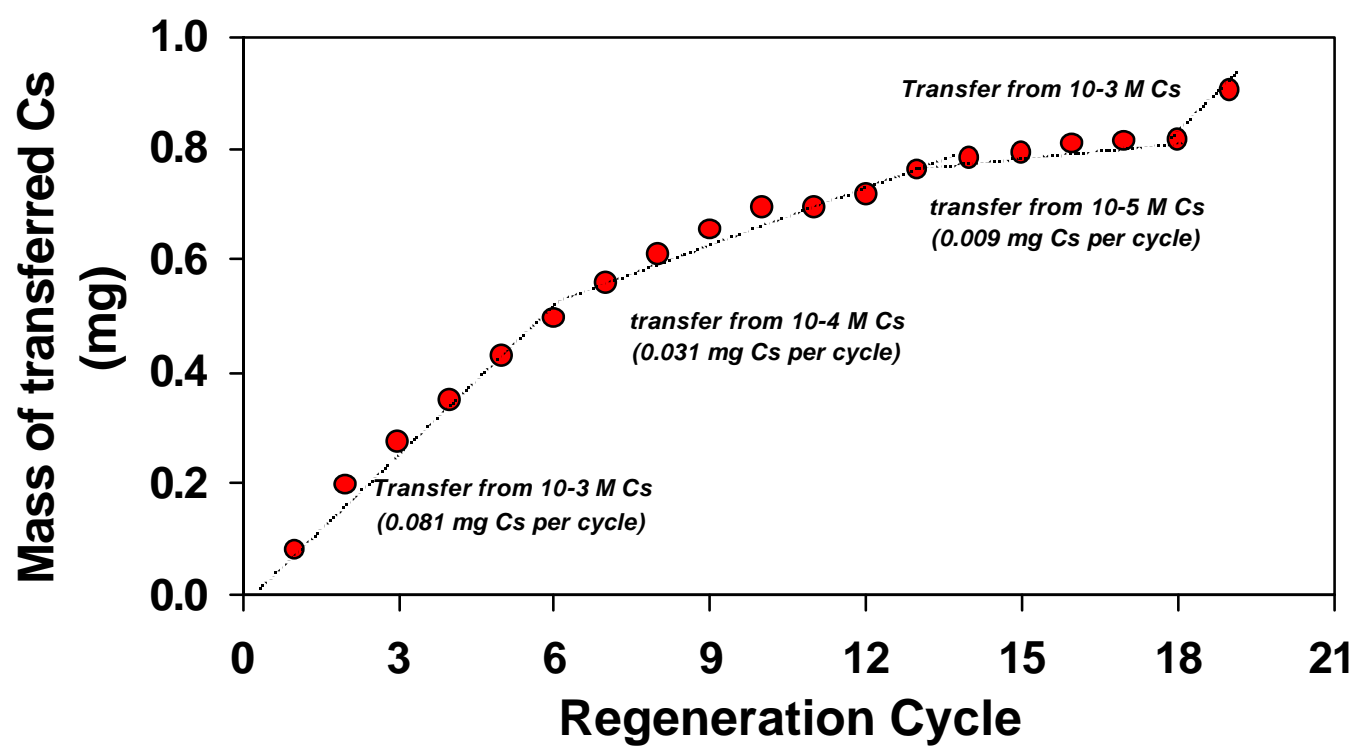

Figure 13. Dependence of Cs transfer on its concentration in the influent. Ni mesh electrode.

In summary, the results of the project were as follows. Sr could be separated from simulated Hanford wastes and concentrated into a relatively small volume of solution by adsorption onto IOCS, followed by acid regeneration of the adsorbent. The advantages of separating $\mathrm{Sr}$ from high-pH, high ionic strength wastes using IOCS include the following:

- IOCS is a selective and efficient adsorbent for $\mathrm{Sr}$ in high $\mathrm{pH}$, high strength wastes.

- IOCS is inexpensive and nontoxic.

- $\mathrm{Ca}, \mathrm{Al}$ and $\mathrm{Cr}$ are not sorbed significantly by IOCS at the $\mathrm{pH}$ of the Hanford wastes, so they do not adversely affect the performance of IOCS or interfere with potential ultimate vitrification of the regenerant.

- IOCS is stable and can be used for many repetitive treatment/regeneration cycles before it needs to be disposed.

- IOCS regeneration is simple and reliable, requiring only a dilute solution of nitric acid. The Sr-containing regenerant may be used repetitively to minimize the ultimate volume of Sr-containing waste needing to be disposed, limited only by the efficiency with which the regenerant can be expelled from the column at the end of the regeneration step or the radiation level of the solution.

- Given the simple composition of the regenerant, its volume can be further decreased by evaporation, or it can be used for vitrification as is. Spent IOCS can also be vitrified or, alternatively, the active coating can be stripped by a strong acid and the sand core can be re-coated.

- Separation of soluble components from liquid waste in packed bed columns is a wellestablished engineering process, so a body of practical experience can be applied in the design and operation of the system.

- The process can be designed to operate automatically, simplifying the operations and reducing radioactive exposure risks to personnel. 
The benefits of electrochemically controlled ion exchange, using metal HCFs to separate

Cs from Hanford waste include the following:

- HCFs are extremely selective for Cs. Their performance is not affected by the presence of massive amounts of sodium salts in the solution.

- EIX dramatically reduces the amount of metal HCFs needed to collect Cs from the waste and thus eases or eliminates problems associated with the long-term storage of Cs-laden HCF solids.

- Cs can be transferred from influents having very high ionic strengths and otherwise complex chemical parameters, into solutions containing only moderate amounts of potassium and cesium salts.

- The Cs can be transferred from influents having low concentrations of Cs to solutions (regenerants) containing Cs concentrations that are larger by many orders of magnitude.

- If necessary, the volume of the regenerant can be further reduced by evaporation. The remaining solids can be easily vitrified.

- The process is controlled solely by external electronic devices; no chemical additions are required.

- The process can be easily automated to reduce risks to personnel.

\section{Perspectives and future research}

Issues that need that to be explored in the future to improve the IOCS-based technology and to facilitate its implementation include the following:

- Examine Sr retention by IOCS in the presence of organic ligands other than EDTA.

- Quantify the effects of competitive complexation of $\mathrm{Sr}, \mathrm{Mg}$ and $\mathrm{Ca}$ in treatment of waste s containing Sr-binding organic ligands.

- Elucidate effects of elevated temperature $\left(40-70^{\circ} \mathrm{C}\right)$ on the performance of IOCS in $\mathrm{Sr}$ separation.

- Quantify short- and long-term radiation effects on IOCS performance.

- Examine the behavior of IOCS in vitrification processes.

- Evaluate the option of re-coating spent IOCS without stripping the initial active layer, or stripping followed by a complete re-coating.

- Evaluate ways to overcome potential operational problems associated with sudden $\mathrm{pH}$ changes, formation of precipitates and clogging.

- Examine ways to monitor the performance of IOCS columns on line to avoid system failure.

- Evaluate the cost of IOCS technology and compare it with that of other processes.

For electrochemically controlled ion exchange, issues that need to be pursued include:

- The stability of the HCF-modified electrodes at high $\mathrm{pH}$ needs to be improved.

- The theoretical constraints and benefits of EIX over conventional cation exchange need to be explored.

- Effects of mass transfer to and from the electrode surface need to be explored.

- The selectivities of the reduced and oxidized forms of HCFs for Cs have to be compared.

- Ways to increase the amount of the active phase in the electrode and decrease the carryover in the pores need to be explored.

- The stability of HCFs in the presence of organic ligands (e.g., EDTA) needs to be addressed. 
- The effects of elevated temperature $\left(40-70^{\circ} \mathrm{C}\right)$ on the performance of $\mathrm{HCFs}$ need to be elucidated.

- Reactivation of HCFs whose electrochemical activity has decayed should be evaluated.

- The cost of EIX technology should be compared with that of other processes.

\section{Relevance, Impact and Technology Transfer}

The scientific knowledge gained in this project is directly relevant to the need to develop efficient and cost-effective technologies to separate radioactive $\mathrm{Sr}$ and Cs isotopes from the other constituents of Hanford tank wastes. The experiments conducted as part of this project focused on the retention of Sr by IOCS and on the benefits provided by electrochemical control of the exchange capacity of hexacyanoferrates. The results suggest that IOCS can achieve virtually complete $\mathrm{Sr}$ removal from high ionic strength, extremely caustic wastes that do not contain organic complexing agents. With appropriate control of the water chemistry, it appears that very good separation of Sr from such solutions can also be achieved using IOCS, even if the solutions contain a strong organic chelating agent (EDTA).

EC-controlled Cs retention can potentially decrease the amount of the exchanger needed to retain Cs and decrease the residual concentration of Cs in the waste. Using EIX, it was possible to separate and transfer Cs from wastes containing as little as $10^{-6} \mathrm{M}$ Cs to regenerant solutions containing up to $10^{-2} \mathrm{M}$ Cs. Without the EC control, no transfer in such conditions was possible.

Ultimately, the impact of this study will be determined by how the results are used. However, the study has provided all the information needed to assess the potential value of $\mathrm{Sr}$ sorption from Hanford wastes by IOCS, and of EC control of HCF-based adsorption of Cs from those wastes. The IOCS-based technology is ready to be transferred to DOE applications. As for the EC controlled HCF technology, more research needs to be carried out before the technology can be practically implemented. Contacts have been established with the groups lead by Michael Lilga at PNNL and Daniel Schwarz at University of Washington, respectively, in an effort to coordinate and prioritize further research.

\section{How will the new scientific knowledge generated in the project improve technologies and cleanup approaches to significantly reduce future costs, schedules and risks and meet DOE compliance parameters?}

Due to the high selectivity of IOCS for Sr, its chemical stability, low cost, ease of preparation and, presumably, lack of problems related to long term storage or vitrification, the use of this material appears to be a highly promising option for $\mathrm{Sr}$ removal from high level liquid tank wastes. Since the regeneration of this material is achieved using an extremely simple routine that generates small volumes of residual waste, on site treatment of large volumes of primary wastes appears feasible. The $\mathrm{Sr}$ in the primary wastes can be separated from the matrix of inert salts, while the regenerant containing ${ }^{90} \mathrm{Sr}$ as virtually the only contaminant of concern can be further processed to reduce its volume. Neither preparation nor handling and use of IOCS are associated with any significant risks to personnel, nor do they require deployment of specialized, costly or difficult-to-operate equipment. Thus, in our opinion, this material is ready for use in pilot scale experiments. We believe that its employment can significantly accelerate the pace and reduce the costs of the on-site treatment of the tank wastes. 
The use of EC-controlled HCFs for Cs separation has great practical promise, but its application requires more work to improve its chemical stability, notably at high pHs. It is also necessary to develop electrodes that overcome limitations associated with mass transfer and capture of the regenerant by the pores in the active material; however, these challenges do not appear to be particularly severe. In the long term, we envision the use of HCF-based electrochemical systems to control Cs in a variety of aqueous media (not necessarily having high $\mathrm{pH}$ ). Since EC control enables regeneration of the hexacyanoferrate exchanger, much benefit is expected due to the significant reduction or complete elimination of any long-term storage of the HCFs.

\section{To what extent does the new scientific knowledge bridge the gap between broad fundamental research that has wide-ranging applications and the timeliness to meet needs- driven applied technology development?}

This study was based on sound scientific principles and, at the same time, was designed to address pressing practical needs. The Sr separation work was a direct extension of previous studies by the principal investigators, extending that work to new target species and extreme solution compositions. The part of this study addressing Cs collection was initiated based on the application of fundamental electrochemical principles to a challenging practical problem. In the work, theoretical analyses of cation exchange equilibria were combined with experimental studies and modeling of electrochemical processes. The work bridged several scientific areas and yielded valuable knowledge for implementing a new technological process. The scientific and technological results of this work can now be applied at pilot or larger scale. They also form a solid foundation for further exploration. The applicability of the results extends beyond the highly specialized application niches investigated experimentally to other issues of potential interest for EMSP programs (e.g., separation of chromium from a variety of wastes using IOCS, separation of Cs from neutral and acidic wastes with EC-controlled HCFs).

What is the project's impact on individuals, laboratories, departments and institutions? Will the results be used? If so, how they will be used, by whom and when?

The impact of the project on the research and teaching activities of the Department of Civil and Environmental Engineering of University of Washington has been notable. First, the principal investigators have been exposed to the pressing remediation needs at DOE sites, notably the Hanford Nuclear site. Although the project has been completed, the investigators will follow up this work by continuing analysis of its results, publishing them in refereed journals and pursuing new and related projects. They have made some contacts with the DOE personnel. Together with the principal investigators, a number of students from this country and abroad (Denmark) have been involved in the project and have been exposed to the realities and quandaries of nuclear remediation.

Several departmental seminars describing this project and related remediation and cleanup problems have been presented. The results will also be used in both teaching and further research projects. In teaching, the modeling results for the $\mathrm{Sr}$ speciation and its changes in response to competition among $\mathrm{Sr}, \mathrm{Ca}$, and $\mathrm{Mg}$ for carbonate and EDTA will be used in classes in aquatic chemistry taught for undergraduate and graduate students. We also plan to incorporate a sequence of lectures related to the nuclear contamination in general, and the problems at the Hanford nuclear site in particular, into the curriculum of the aquatic 
chemistry course offered for undergraduates, and possibly into courses in physical/ chemical water treatment technologies.

As for the University of Washington in general, several projects funded by the EMSP programs have been run at this institution. The impact of these projects on the research and teaching at the university is difficult to evaluate, but there is little doubt that the level of exposure of both the faculty and students to the problems of DOE sites is now incomparably larger than prior to the onset of these projects.

Are larger-scale trials warranted? What difference has the project made? Now that the project is complete, what new capacity, equipment or expertise has been developed?

The PIs feel strongly that larger scale trials are warranted for the IOCS-based separation technology. For EIX, expanded trials will be warranted following further improvement of the electrode design.

This study showed that a relatively simple, low-cost product (IOCS) can perform remove $\mathrm{Sr}$ from solution very well under extremely challenging conditions (very high concentrations of hydroxide and nitrate, presence of a variety of competing metal cations). Based on this result, on- site treatment of liquid tank wastes can be substantially simplified and accelerated, while the generation of material that requires subsequent vitrification and long-term storage can be sharply curtailed. Also, the EIX technology tested in this project, although not yet practically deployable at full-scale, has demonstrated important benefits (e.g., elimination of the need to store $\mathrm{HCF}$ solids, ability to concentrate Cs in a small volume). We believe that EIX-related technologies, not necessarily utilized solely in the nuclear cleanup, will be developed. Third, we have started studies of the intrinsic structure and stability of iron oxide and HCF products in collaboration with our colleagues at University of Illinois at UrbanaChampaign using synchrotron equipment located at Brookhaven National Laboratory. Thus, we have acquired a capacity and expertise to carry out joint studies that combine the exploration of novel treatment approaches with chemical equilibrium modeling and sophisticated material science research. This combination will be very important for us, as well as for research in the broader field of nuclear and environmental remediation in the future.

\section{How have the scientific capabilities of collaborating scientists been improved?}

This has been answered.

\section{How has this research advanced our understanding in the area?}

Adsorption at extremely high $\mathrm{pH}$ has been studied only to a very limited extent previously. The results for the relative adsorbability of $\mathrm{Sr}, \mathrm{Ca}$, and $\mathrm{Cr}$ at $\mathrm{pH}>14$ provide support for the hypothesis that, while singly-hydrolyzed metals $(\mathrm{MeOH})$ can sorb, metals that have lost more than one proton from their inner hydration sphere cannot. With respect to EIX, although this technique had been proposed before the project was started, virtually nothing had been done to examine the performance of this method theoretically, and little to demonstrate the actual transfer and concentration of cesium using HCF-modified electrodes. This research generated an extensive amount of data to demonstrate the actual performance of this technology, and provided results needed to quantify the efficiency of the electrochemical control and to understand better conditions under which its use is practical. 
In addition to that, we have initiated both a theoretical analysis of this technology and further in-depth exploration of the fundamental properties of this important class of materials using advanced experimental methods.

What additional scientific or other hurdles must be overcome before the results of this project can be successfully applied to DOE Environmental Management Problems?

Have any other government agencies expressed interest in the project? Please provide contact information.

Regarding Sr removal by IOCS, contact has been made with Daniel McCabe and Major Thompson at SRS. One set of batch tests evaluating the partitioning coefficient of Sr when exposed to IOCS was conducted, and the results were not highly encouraging. Considering the vast amount of data we have collected that shows that IOCS can remove Sr effectively from simulated supernatant solutions and the 'tweaking' of the water chemistry that must be done before the ideal conditions for such removal can be identified, and considering the enormous potential value of using IOCS in the way described in this report, we feel strongly that the potential for using IOCS should not be ruled out based on a single set of very preliminary and limited tests. It appears that DOE personnel do not share this opinion.

\title{
Project productivity
}

The project accomplished its main objectives. The only modification to the work plan was that, because preliminary tests indicated that the adsorption/ microfiltration process for treating Sr was less effective than adsorption onto IOCS in columns, the effort on the former process was reduced and that on the latter process was expanded in the latter portions of the research. The research was completed on time, although the final project report is being submitted a few months later than scheduled.

\section{Personnel Supported}

\author{
Mark Benjamin \\ Gregory Korshin \\ Birgitte Ostegaard-Hansen \\ Henrik Green-Pedersen \\ Chi-Wang Li \\ Mark Jensen \\ Pierre Kwan
}

\section{Publications}

One article describing the project results has been published in peer-reviewed scientific journals, and four others are in preparation. The citations or other descriptions of these articles are provided below:

a. Published in peer-reviewed journal:

Green-Pedersen, H., G.V.Korshin (1999). Separation of cesium from high ionic strength solutions using a cobalt hexacyanoferrate-modified graphite electrode.

Environmental Science and Technology, 33 (15): 2633-2637. 
b. Manuscripts in preparation for submittal to peer-reviewed journals

"Sorption of Sr by IOCS from Simulated Hanford Tank Wastes." This manuscript will describe the removal of $\mathrm{Sr}$ from the simulated wastes, regeneration of the media, the effect of EDTA and $\mathrm{Ca}$ on Sr adsorption, and the modeling results for the potential use of $\mathrm{Mg}$ instead of Ca to overcome the adverse effect of EDTA on Sr sorption.

"Theoretical analysis of the performance of hexacyanoferrate active phases for separation of cesium using electrochemically-controlled cation exchange". This paper will examine the benefits, drawbacks and limitations of the use of EIX for cesium separation and preconcentration. Based on numerical modeling of the equilibria involving the relevant solid phases and solutions, this paper will establish the limits of Cs removal using the EIX or solid HCF phases, and the effects HCF selectivity and electrochemical control on the performance of EIX.

"Comparison of the performance of various HCF-modified electrodes for cesium separation and transfer using EIX". This paper will present experimental data that show the benefits of EIX control for removal and preconcentration of Cs. It will also discuss the instability of the HCF phases and methods to reactivate them.

"EXAFS studies of the incorporation of hydrated cesium ions into hexacyanoferrate solids". To determine the theoretical limits of the stability of HCFs in highly alkaline solutions, it is necessary to explore the nature of the interactions between cesium cations and the solid. This paper will describe experimental data relevant to the chemical state of cesium cations in the HCF matrix and will discuss whether the incorporated ions undergo dehydration and other structural transformations.

\section{Interactions}

The Principal Investigators have presented the results of the research at DOE meetings in Chicago (1998), Albuquerque (1999), and Atlanta (2000). Interactions with DOE personnel at these meetings have been useful and productive in some cases, although follow-up has not been as easy or as successful as we had hoped.

\section{Transitions}

We are still seeking appropriate ways to facilitate transition of the research results to end users.

\section{Patents}

No inventions or patentable materials were generated by this project. 


\section{Future Work}

As noted above, the IOCS-based technology is ready to be transferred to DOE applications. As for the EC controlled HCF technology, more research needs to be carried out before the technology can be practically implemented. 